\title{
DISCRETIZATION OF SECOND-ORDER VARIATIONAL SYSTEMS
}

\author{
MICHAL FEČKAN \\ (Communicated by Charles C. Pugh)
}

\begin{abstract}
The existence of periodic orbits of the discretization of second-order variational systems is studied both for varying step of discretization and for a fixed small one.
\end{abstract}

\section{INTRODUCTION}

The purpose of this paper is to study the existence of periodic orbits of the mapping

$$
\phi_{h}:(x, y) \rightarrow\left(2 \cdot x-y+h^{2} \cdot f(x), x\right),
$$

where $(x, y) \in R^{m} \times R^{m}, h>0, f: R^{m} \rightarrow R^{m}$ is $C^{1}$-smooth, $f=\operatorname{grad} g$. We note that (1.1) presents the Euler-discretization of the equation

$$
z^{\prime \prime}=f(z)
$$

with the step of magnitude $h$. Indeed, the discretization of (1.2) has the form

$$
z_{n+1}-2 \cdot z_{n}+z_{n-1}=h^{2} \cdot f\left(z_{n}\right)
$$

and putting $z_{n}=x_{n}, z_{n-1}=y_{n}$ we have

$$
\begin{aligned}
& x_{n+1}=2 \cdot x_{n}-y_{n}+h^{2} \cdot f\left(x_{n}\right), \\
& y_{n+1}=x_{n} .
\end{aligned}
$$

Hence periodic orbits of $\phi_{h}$ are periodic orbits of the Euler-discretization of (1.2) with the step $h$.

In $\S 2$ we investigate local behaviours of $(1.1)$. We shall assume that $f(0)=$ $0, D f(0)$ has eigenvalues $-\alpha_{1}^{2} \leq \cdots \leq-\alpha_{m-s}^{2}<0<\beta_{1}^{2} \leq \cdots \leq \beta_{s}^{2}$. By a result of Berger [3] we know that the system (1.2) has $m-s$ one-parameter families of periodic solutions with periods tending to $2 \pi / \alpha_{i}$ and with amplitudes tending to 0 . The next natural question arises: whether the mapping (1.1) has, for fixed $h, m-s$ families of invariant circles with amplitudes tending to 0 and rotation numbers tending to $2 \pi / \alpha_{i} h, \ldots, 2 \pi / \alpha_{m-s} h$. By the remarks below the proof of Theorem 2.1 of this paper, we know that for $h$ small the mapping $\phi_{h}$ has at 0 a center manifold of dimension $2(m-s)$ corresponding to $2(m-s)$ nonreal

Received by the editors June 12, 1991.

1991 Mathematics Subject Classification. Primary 58F08; Secondary 58F22. 
eigenvalues on the unit circle and stable and unstable manifolds of dimensions $s$. But easy computations show that the same property holds for (1.2). Indeed, the linearization of (1.2) at 0 has the eigenvalues $\pm l \cdot \alpha_{1}, \ldots, \pm l \cdot \alpha_{m-s}$ and $\pm \beta_{1}, \ldots, \pm \beta_{s}$. This correspondence may support a positive answer of the question. The author is not able to give this positive answer, because it seems that this problem is very difficult. Instead of this, we shall vary $h$ and thus $h$ will be a bifurcation parameter. We show (see Theorem 2.1) that there is an interval $(0, \omega)$ such that the set of bifurcation values of $h$ of periodic orbits of (1.1) near 0 is dense in this interval. Of course, this result is consistent with a positive answer to the question, because the existence of the abovementioned families of invariant circles of (1.1) immediately implies this result for $\omega$ small. Actually, our proof shows that we can take $\omega=2 / \alpha_{m-s}$, and hence our result is not restricted to $h$ small. In the last part of this section we fix $h$ and globally study the existence of periodic orbits of (1.1). We are also stimulated by a similar result of Hamiltonian systems [7, 2]. In these papers the authors studied (1.2) for $f=f(z, t)$ depending also on $t$ and $f$ is $2 \pi$ periodic in $t$. They assumed that 0 is a trivial solution of such a system and $f$ is asymptotically linear at infinity. Under some conditions they showed the existence of a nontrivial periodic solution. Similarly, we shall assume that $f$ has only a finite number of zeros and $f$ is asymptotically linear at infinity. Then under some conditions we show the existence of an infinite number of periodic orbits of (1.1) for $h$ small fixed, with minimal periods tending to infinity.

On the other hand, we note that the map $\phi_{h}$ can be considered as a higher dimensional version of the Hénon map [6] and, moreover, $\phi_{h}$ is volume preserving. There is a relation between area preserving twist maps [1] and $\phi_{h}$ as well. Indeed, following $[1$, p. 355] we put

$$
h(x, z)=-|x-z|^{2}-h^{2} \cdot g(z),
$$

and define a map $F$ as $F\left(x_{0}, y_{0}\right)=\left(x_{1}, y_{1}\right)$ iff

$$
y_{0}=\frac{\partial}{\partial x} h\left(x_{0}, x_{1}\right), \quad y_{1}=-\frac{\partial}{\partial x_{1}} h\left(x_{0}, x_{1}\right) \text {. }
$$

Then

$$
\begin{aligned}
& y_{0}=x_{1}-x_{0} \\
& y_{1}=x_{1}-x_{0}+h^{2} \cdot f\left(x_{1}\right)
\end{aligned}
$$

i.e.,

$$
\begin{aligned}
& x_{1}=y_{0}+x_{0}, \\
& y_{1}=y_{0}+h^{2} \cdot f\left(x_{0}+y_{0}\right) .
\end{aligned}
$$

Since $y_{k}=x_{k+1}-x_{k}$, we have

$$
x_{k+1}-2 \cdot x_{k}+x_{k-1}=h^{2} \cdot f\left(x_{k}\right) \text {. }
$$

Thus the map $F$ generates the map $\phi_{h}$. We point out that our approach is similar to [1], since we shall also seek critical points of a functional defined similarly as in [1, p. 354]. Critical points of this functional are periodic orbits of $F$. For finding critical points we use a bifurcation theorem for variational problems [4] in a proof of Theorem 2.1 and Theorem 1.2 from [7] for proving Theorem 3.1 in this paper. 
Finally, let us consider the mapping on $R^{2}$

$$
\omega_{h}:\left\{\begin{array}{l}
x_{1}=x_{0}+y_{0}, \\
y_{1}=y_{0}+h^{2} \cdot f\left(x_{0}+y_{0}\right),
\end{array}\right.
$$

where $f(0)=0, f \in C^{4}, f^{\prime}(0)<0$, and $f$ is 1-periodic. Note that

$$
\left(\begin{array}{cc}
0 & 1 \\
1 & -1
\end{array}\right) \cdot \phi_{h}=\omega_{h} \cdot\left(\begin{array}{cc}
0 & 1 \\
1 & -1
\end{array}\right)
$$

We can consider $\omega_{h}$ as a map on $S^{1} \times R$. Applying the twist theorem of Moser [6] , we see that most of invariant curves of the form $S^{1} \times\{r\}$ of $\omega_{0}$ survive for $h$ small. Thus, the existence of a family of invariant curves around $(0,0)$ approximating solutions of $x^{\prime \prime}=f(x)$ is imposible. We note that $(0,0)$ is an elliptic fixed point of $\omega_{h}$ for $h$ small. Thus if $\omega_{h}$ is sufficiently smooth and $(0,0)$ is a nondegenerate elliptic fixed point, then the dynamics of $\omega_{h}$ are well known on some small open neighbourhood $U_{h}$ of $(0,0)$. Unfortunately $U_{h}$ must shrink to the $y$-axis as $h \rightarrow 0$. The advantage of our result is that we can predict some periodic orbits of $\omega_{h}$ for $h$ varying by using only the linearizations of $\omega_{h}$ at $(0,0)$.

\section{LOCAL EXISTENCE}

We assume that $f(0)=0$ and $D f(0)$ has eigenvalues $-\alpha_{1}^{2} \leq \cdots \leq-\alpha_{m-s}^{2}<$ $0<\beta_{1}^{2} \leq \cdots \leq \beta_{s}^{2}$. The following theorem shows us the existence of periodic orbits of $(1.1)$ near $(0,0)$.

Theorem 2.1. For each $h \in\left\langle 0,2 / \alpha_{m-s}\right\rangle$ and $\delta>0$ there is a sequence $\left\{h_{n}\right\}_{1}^{\infty}$ $\subset\left\langle 0,2 / \alpha_{m-s}\right\rangle$ such that $h_{n} \rightarrow h$ and $\phi_{h_{n}}$ has a nontrivial periodic orbit $\left\{y_{1}, \ldots, y_{n}\right\}$ satisfying $\sup _{i}\left|y_{i}\right|<\delta$. Hence the set

$\left\{h \in\left\langle 0,2 / \alpha_{m-s}\right\rangle \mid \phi_{h}\right.$ possesses an orbit with the minimal period $n \geq n_{0}$ and amplitude smaller than $\delta\}$

is dense in $\left\langle 0,2 / \alpha_{m-s}\right\rangle$ for each $n_{0}>0, \delta>0$ fixed. (By the trivial orbit we mean $y_{i}=0, i=1, \ldots, n$.)

Proof. Let $\left(z_{1}, \ldots, z_{n}\right)$ be a periodic orbit of (1.3). Then

$$
z_{2}-2 \cdot z_{1}+z_{n}=h^{2} \cdot f\left(z_{1}\right)
$$

$$
\begin{aligned}
z_{n}-2 \cdot z_{n-1}+z_{n-2} & =h^{2} \cdot f\left(z_{n-1}\right) \\
z_{1}-2 \cdot z_{n}+z_{n-1} & =h^{2} \cdot f\left(z_{n}\right) .
\end{aligned}
$$

We put as in [5]

$$
\begin{gathered}
D:\left(R^{m}\right)^{n} \rightarrow\left(R^{m}\right)^{n}, \quad F:\left(R^{m}\right)^{n} \rightarrow\left(R^{m}\right)^{n}, \\
D\left(z_{1}, \ldots, z_{n}\right)=\left(z_{2}-2 \cdot z_{1}+z_{n}, \ldots, z_{1}-2 \cdot z_{n}+z_{n-1}\right), \\
F\left(z_{1}, \ldots, z_{n}\right)=\left(f\left(z_{1}\right), \ldots, f\left(z_{n}\right)\right),
\end{gathered}
$$

and hence (2.1) has the form

$$
D z=h^{2} \cdot F(z), \quad z=\left(z_{1}, \ldots, z_{n}\right) .
$$


We note that $D$ is symme:ric and $F$ has a gradient form. Hence (2.2) also has a variational form. By our assumption, $z=0$ is a solution of (2.2). We compute the linearization of (2.2) at $z=0$,

$$
D v=h^{2} \cdot D F(0) v,
$$

where $D F(0)=(D f(0), \ldots, D f(0))$. Since $D f(0)$ is symmetric by using a change of variables, we can assume that $D f(0)$ is diagonal possessing the form

$$
D f(0)=\left(\begin{array}{cccccccc}
-\alpha_{1}^{2} & 0 & \cdot & \cdot & \cdot & \cdot & \cdot & \cdot \\
\cdot & \cdot & \cdot & \cdot & \cdot & \cdot & \cdot & \cdot \\
\cdot & \cdot & \cdot & \cdot & \cdot & \cdot & \cdot & \cdot \\
\cdot & \cdot & 0 & -\alpha_{m-s}^{2} & 0 & \cdot & \cdot & \cdot \\
\cdot & \cdot & \cdot & 0 & \beta_{1}^{2} & 0 & \cdot & \cdot \\
\cdot & \cdot & \cdot & \cdot & \cdot & \cdot & \cdot & \cdot \\
\cdot & \cdot & \cdot & \cdot & \cdot & \cdot & \cdot & \cdot \\
\cdot & \cdot & \cdot & \cdot & \cdot & \cdot & 0 & \beta_{s}^{2}
\end{array}\right) .
$$

We are interested in eigenvalues of (2.3). It is clear that for this purpose it is sufficient to find the solution $\lambda$ of the equation

$$
z_{2}-2 \cdot z_{1}+z_{n}=h^{2} \cdot \gamma \cdot z_{1}+\lambda \cdot z_{1}
$$

$$
z_{1}-2 \cdot z_{n}+z_{n-1}=h^{2} \cdot \gamma \cdot z_{n}+\lambda \cdot z_{n},
$$

where $\gamma \in\left\{-\alpha_{1}^{2}, \ldots, \beta_{s}^{2}\right\}, z_{i} \in R$. But we know from [5] that the left side matrix of (2.4) has the eigenvalues

$$
\left\{-4 \cdot \sin ^{2}(\pi / n) \cdot j \mid j=0, \ldots, n-1\right\} .
$$

Hence $\lambda \in\left\{-4 \cdot \sin ^{2}(\pi / n) \cdot j-h^{2} \cdot \gamma \mid j=0, \ldots, n-1\right\}$. But this means that if we consider $h$ as a parameter in (2.1) then each value of $h$ such that $-4 \cdot \sin ^{2}(\pi / n) \cdot j-h^{2} \cdot \gamma=0$ is a bifurcation point (see [4]). Hence $\mathscr{M}_{n}=$ $\left\{2 \cdot \sin (\pi / n) \cdot j / \alpha_{i} \mid i=1, \ldots, s, j=0, \ldots, n-1\right\}$ are bifurcation points of (2.1). Finally, we note that $\bigcup \mathscr{M}_{n}$ is dense in $\left\langle 0,2 / \alpha_{m-s}\right\rangle$ and the proof is finished.

We compute the linearization of (1.1) at $x=y=0$

$$
\left(x_{1}, y_{1}\right) \rightarrow\left(2 \cdot x_{1}-y_{1}+h^{2} \cdot D f(0) x_{1}, x_{1}\right),
$$

and since $D f(0)$ is diagonal, (2.5) has a coordinate form

$$
\left(x_{1}, y_{1}\right) \rightarrow\left(2 \cdot x_{1}-y_{1}+h^{2} \cdot \gamma \cdot x_{1}, x_{1}\right),
$$

where $\gamma \in\left\{-\alpha_{1}^{2}, \ldots, \beta_{s}^{2}\right\}$. The eigenvalues of (2.6) are

$$
\lambda_{1 / 2}=\left(2+h^{2} \cdot \gamma \pm h \cdot \sqrt{h^{2} \cdot \gamma^{2}+4 \cdot \gamma}\right) / 2 .
$$

We see that for $\gamma>0,\left|\lambda_{1 / 2}\right| \neq 1$ and for $\gamma<0$ and for $h>2 \cdot \sqrt{-1 / \gamma}$, $\left|\lambda_{1 / 2}\right| \neq 1$, but for $\gamma<0, h \in(0,2 \cdot \sqrt{-1 / \gamma}), \quad\left|\lambda_{1 / 2}\right|=1$. Thus for $h \in\left(0,2 / \alpha_{m-s}\right\rangle$ the linearization of $(1.1)$ at $x=y=0$ has an eigenvalue with the absolute value 1 and for $h>2 / \alpha_{m-s}$ it has not such an eigenvalue. Hence $x=y=0$ is a hyperbolic fixed point of $\phi_{h}$ only for $h>2 / \alpha_{m-s}$, and this implies 
Theorem 2.2. There is $\delta>0$ and a neighbourhood $U$ of $(0,0) \in R^{2 m}$ for each $h_{0}>2 / \alpha_{m-s}$ such that for each $h \in\left(h_{0}-\delta, h_{0}+\delta\right)$ the map $\phi_{h}$ has no nontrivial periodic orbit in $U$.

Summing up we can state that if $h$ varies in $\left(2 / \alpha_{m-s}, \infty\right)$ then the dynamics of $\phi_{h}$ near $(0,0) \in R^{2 m}$ does not change, but for $h \in\left(0,2 / \alpha_{m-s}\right\rangle$ there are bifurcations of periodic orbits in arbitrary small neighbourhood of $(0,0)$.

\section{A global Result}

In the previous section we study $\phi_{h}$ for varying $h$. Now we fix $h>0$ and study the existence of periodic orbits of $\phi_{h}$ in $R^{m} \times R^{m}$. We must solve (2.2) for the existence of n-periodic orbits. We assume for $f$

(i) $f$ has only zero points $x_{1}, x_{2}, \ldots, x_{k}$,

(ii) $D f\left(x_{i}\right), i=1, \ldots, k$, have nonzero eigenvalues,

(iii) $|f(x)-A x| /|x| \rightarrow 0$ as $|x| \rightarrow 0$ for a symmetric matrix $A \in \mathscr{L}\left(R^{m}\right)$ with $\operatorname{det} A \neq 0$.

Since $D f\left(x_{i}\right)$ and $A$ are symmetric, they have only real nonzero eigenvalue, and let $\left\{d_{i}^{p}, \ldots, d_{m}^{p}\right\}$ and $\left\{a_{1}, \ldots, a_{m}\right\}$ be eigenvalues of $D f\left(x_{p}\right)$ and $A$, respectively, for $p=1, \ldots, k$.

Theorem 3.1. Let us assume

$$
\sum_{a_{j}<0} \sqrt{-a_{j}} \neq \sum_{d_{j}^{p}<0} \sqrt{-d_{j}^{p}}
$$

for each $p=1, \ldots, k$. Then for $h>0$ sufficiently small there is a sequence of natural numbers $\left\{n_{i}\right\}_{0}^{\infty}$ such that $n_{i}<n_{i+1}, n_{i} \rightarrow \infty$ as $i \rightarrow \infty$ and $\phi_{h}$ has a periodic orbit with the minimal period $n_{i}$ for each $i$. (If $\left\{a_{i}, a_{i}<0\right\}=\varnothing$ then we put $\sum_{a_{i}<0}=0$.)

Proof. We shall apply the following theorem.

Theorem [7]. Let $a: R^{m} \rightarrow R$ be a $C^{2}$-function satisfying

$$
\left|\nabla a(x)-A_{\infty} x\right| /|x| \rightarrow 0 \text { as }|x| \rightarrow \infty
$$

for a symmetric nonsingular matrix $A_{\infty} \in \mathscr{L}\left(R^{m}\right)$. Suppose that a has critical points $\tilde{x}_{1}, \ldots, \tilde{x}_{k}$ and all of them are nondegenerate. If $M^{+}\left(a^{\prime \prime}\left(\tilde{x}_{i}\right)\right) \neq M^{+}\left(A_{\infty}\right)$ for each $i$ then a has another critical point. Here $a^{\prime \prime}$ is the Hessian and $M^{+}(B)$ is the positive Morse index of the symmetric matrix $B$.

Let $n$ be odd. We have for our case

$$
\begin{gathered}
a(z)=\frac{\langle D z, z\rangle}{2}-h^{2} \cdot \sum_{i=1}^{n} g\left(z_{i}\right), \\
\tilde{x}_{i}=\left(x_{i}, \ldots, x_{i}\right), \quad A_{\infty}(z)=D-h^{2} \cdot\left(A z_{1}, \ldots, A z_{n}\right) .
\end{gathered}
$$

Note that $\operatorname{grad} g=f$. We shall compute $M^{+}\left(a^{\prime \prime}\left(\tilde{x}_{i}\right)\right)$. Since $a^{\prime \prime}\left(\tilde{x}_{i}\right)=D-h^{2}$. $D F\left(\tilde{x}_{i}\right)$ where $D F\left(\tilde{x}_{i}\right)=\left(D f\left(x_{i}\right), \ldots, D f\left(x_{i}\right)\right), D f\left(x_{i}\right)$ can be considered in a diagonal form having the eigenvalues $d_{1}^{i}, \ldots, d_{m}^{i}$. We can show, as in the above proof, that $a^{\prime \prime}\left(\tilde{x}_{i}\right)$ has eigenvalues

$$
\left\{-4 \cdot \sin ^{2} \frac{\pi}{n} \cdot j-h^{2} \cdot d_{s}^{i} \mid j=0, \ldots, \frac{(n-1)}{2}, s=1, \ldots, m\right\} .
$$


Similarly, $A_{\infty}$ has eigenvalues

$$
\left\{-4 \cdot \sin ^{2} \frac{\pi}{n} \cdot j-h^{2} \cdot a_{i} \mid j=0, \ldots, \frac{(n-1)}{2}, i=1, \ldots, m\right\} \text {. }
$$

First of all, we must ensure that $a^{\prime \prime}\left(\tilde{x}_{i}\right)$ and $A_{\infty}$ are nonsingular, i.e.,

$$
-4 \cdot \sin ^{2} \frac{\pi}{n} \cdot j-h^{2} \cdot d_{s}^{i} \neq 0, \quad-4 \cdot \sin ^{2} \frac{\pi}{n} \cdot j-h^{2} \cdot a_{i} \neq 0 .
$$

Hence it must hold for $0<h$ small and $d_{s}^{i}<0, a_{i}<0$

$$
\begin{array}{ll}
\frac{j}{n} \neq \arcsin h \cdot \sqrt{\frac{-d_{s}^{i}}{2}} \cdot \frac{1}{\pi}, & j=0, \ldots, \frac{(n-1)}{2}, \\
\frac{j}{n} \neq \arcsin h \cdot \sqrt{-a_{i}} \cdot \frac{1}{\pi}, & j=0, \ldots, \frac{(n-1)}{2} .
\end{array}
$$

We put $\tilde{d}_{s}^{i}=\arcsin h \cdot \sqrt{-d_{s}^{i}} / 2 \cdot \frac{1}{\pi}, \tilde{a}_{i}=\arcsin h \cdot \sqrt{-a_{i}} / 2 \cdot \frac{1}{\pi}$ for $d_{s}^{i}<0, a_{i}<0$. Now it is clear that we can find a sequence of prime numbers $\left\{n_{i}\right\}_{0}^{\infty}$ such that the relation (3.1) holds with $n=n_{i}$ and $n_{i} \rightarrow \infty$ as $i \rightarrow \infty$. Then for $n=n_{b}$

$$
\begin{aligned}
M^{+}\left(a^{\prime \prime}\left(\tilde{x}_{i}\right)\right)=2 & \cdot \#\left\{-4 \cdot \sin ^{2} \frac{\pi}{n_{b}} \cdot j-h^{2} \cdot d_{s}^{i}>0 \mid \frac{\left(n_{b}-1\right)}{2} \geq j>0,\right. \\
& s=1, \ldots, m\} \\
& +\#\left\{d_{s}^{i}<0 \mid s=1, \ldots, m\right\}, \\
M^{+}\left(A_{\infty}\right)=2 & \cdot \#\left\{-4 \cdot \sin ^{2} \frac{\pi}{n_{b}} \cdot j-h^{2} \cdot a_{s}>0 \mid \frac{\left(n_{b}-1\right)}{2} \geq j>0,\right. \\
& +\#\left\{a_{s}<0 \mid s=1, \ldots, m\right\},
\end{aligned}
$$

since $-4 \cdot \sin ^{2}\left(\pi / n_{b}\right) \cdot j, 0<j \leq\left(n_{b}-1\right) / 2$, and 0 have the geometric multiplicity 2 and 1 respectively, in $D$ for $m=1$ (see [5]), and \# denotes cardinality.

Hence

$$
\begin{aligned}
M^{+}\left(a^{\prime \prime}\left(\tilde{x}_{i}\right)\right)= & 2 \cdot \#\left\{\frac{j}{n_{b}}<\tilde{d}_{s}^{i} \mid 0<j \leq \frac{\left(n_{b}-1\right)}{2}, s=1, \ldots, m\right\} \\
& +\#\left\{d_{s}^{i}<0 \mid s=1, \ldots, m\right\}, \\
M^{+}\left(A_{\infty}\right)= & 2 \cdot \#\left\{\frac{j}{n_{b}}<\tilde{a}_{s} \mid 0<j \leq \frac{\left(n_{b}-1\right)}{2}, s=1, \ldots, m\right\} \\
& +\#\left\{a_{s}<0 \mid s=1, \ldots, m\right\} .
\end{aligned}
$$

For $h$ small $\tilde{d}_{s}^{i}<\frac{1}{2}, \tilde{a}_{i}<1$ and thus,

$$
\begin{aligned}
M^{+}\left(a^{\prime \prime}\left(\tilde{x}_{i}\right)\right) & =2 \cdot \sum_{d_{s}^{i}<0}\left(\left[\tilde{d}_{s}^{i} \cdot n_{b}\right]+\frac{1}{2}\right), \\
M^{+}\left(A_{\infty}\right) & =2 \cdot \sum_{a_{i}<0}\left(\left[\tilde{a}_{i} \cdot n_{b}\right]+\frac{1}{2}\right)
\end{aligned}
$$


$([x]$ is the integer part of $x)$. We compute

$$
\begin{aligned}
M^{+}\left(a^{\prime \prime}\left(\tilde{x}_{i}\right)\right)-M^{+}\left(A_{\infty}\right)=2 \cdot n_{b} \cdot h \cdot & \left(\sum_{d_{s}^{i}<0} \frac{\arcsin h \cdot \sqrt{-d_{s}^{i}} / 2 \cdot 1 / \pi}{h}\right. \\
& \left.-\sum_{a_{i}<0} \frac{\arcsin h \cdot \sqrt{-a_{i}} / 2 \cdot 1 / \pi}{h}\right)+C,
\end{aligned}
$$

where $|C| \leq 4 \cdot m$. Now using $\lim _{h \rightarrow 0}(\arcsin h \cdot d) / h=d$ and our assumption concerning $d_{s}^{i}$ and $a_{i}$, we see that for $h>0$ small fixed and $n_{b}$ large

$$
M^{+}\left(a^{\prime \prime}\left(\tilde{x}_{i}\right)\right) \neq M^{+}\left(A_{\infty}\right), \quad i=1, \ldots, k .
$$

But this means that $a$ has a nontrivial critical point, i.e., $\phi_{h}$ possesses an orbit with the minimal period $n_{b}$. Note that $n_{b}$ is a large prime number. This finishes the proof.

\section{ACKNOWLEDGMENT}

The author thanks the referee for a careful reading of the manuscript.

\section{REFERENCES}

1. S. B. Angenent, The periodic orbits of an area preserving twist map, Comm. Math. Phys. 115 (1988), 353-374.

2. V. Benci, A new approach to the Morse-Conley theory, Recent Advances in Hamiltonian Systems (F. D. Antonie and B. D. Onofrion, eds.), World Scientific, Singapure, 1986, pp. $1-52$.

3. M. S. Berger, Nonlinearity and functional analysis, Academic Press, New York.

4. S. N. Chow and R. Lauterbach, A bifurcation theorem for critical points of variational problems, Nonlinear Anal. 12 (1988), 51-61.

5. M. Fečkan, Discretization in the method of averaging, Proc. Amer. Math. Soc. 113 (1991), 1105-1113.

6. J. Guckenheimer and P. Holmes, Nonlinear oscilations, dynamical systems, and bifurcations of vector fields, Springer-Verlag, New York.

7. S. Li and J. Q. Liu, Morse theory and asymptotic linear Hamiltonian systems, J. Differential Equations 78 (1989), 53-73.

Mathematical Institute, Slovak Academy of Sciences, Štefánikova 49, Bratislava, Czechoslovakia 